\title{
Nutritional and sensory characterization of full fat and partially defatted peanut soy milk yoghurt
}

\author{
Kpodo Mawunyo Kwasi Fidelis ${ }^{1, ~ *, ~ A f o a k w a ~ O h e n e ~ E m m a n u e l ~}{ }^{2}$, Amoa Bediako Betty ${ }^{2}$, \\ Saalia Kwesi Firibu² \\ ${ }^{1}$ Department of Hospitality and Tourism Management, Ho Polytechnic, P.O. Box HP 217, Ho, Ghana \\ ${ }^{2}$ Department of Nutrition and Food Science, University of Ghana, P. O. Box LG 134, Legon-Accra, Ghana \\ Email address: \\ fideliskpodo@yahoo.com (F. M. K. Kpodo)
}

\section{To cite this article:}

Kpodo Mawunyo Kwasi Fidelis, Afoakwa Ohene Emmanuel, Amoa Bediako Betty, Saalia Kwesi Firibu. Nutritional and Sensory Characterization of Full Fat and Partially Defatted Peanut Soy Milk Yoghurt. International Journal of Nutrition and Food Sciences. Vol. 3, No. 3, 2014, pp. 187-193. doi: 10.11648/j.jinfs.20140303.19

\begin{abstract}
Yoghurt produced by fermenting milk from peanut and soy milk are considered to have poor sensory attributes due to the off-flavours legumes generate in food products. Improvement in flavour requires a combination of treatments. This study employed three treatments (thermal, chemical and microbial) to develop full fat peanut-soy milk yoghurt (FPSY) and partially defatted peanut-soy milk yoghurt (DPSY). Proximate analysis and consumer studies were carried out on the 10 FPSY and 10 DPSY formulations developed using a three component constraint mixture design. Balanced Incomplete Block Design (BIBD) was used to assign samples to consumers and the optimized formulations were validated. Samples of the FPSY were high in crude protein and fat whereas the DPSY formulations were high in carbohydrate and total solids. Consumers preferred more soy milk in their full fat vegetable milk yoghurts but preferred more cow milk in their low fat vegetable milk yoghurt. FPSY and DPSY formulations with the most preferred sensory attributes were 0.68 Soy milk, 0.25 Peanut milk and 0.07 Cow milk; and 0.65 Soy milk, 0.22 defatted peanut milk and 0.13 cow milk respectively.
\end{abstract}

Keywords: Yoghurt, Peanut Milk, Soy Milk, Balanced Incomplete Block Design, Consumer Studies

\section{Introduction}

Peanut and soy based products have been considered to have poor sensory characteristics due to the beany and other off-flavours they generate in food products that contain them $[1,2]$. Acceptability ratings of these products have been significantly lower than the traditional dairy products. Nonetheless food scientists are still faced with the challenge of formulating foods that are appealing and acceptable to consumers, but still contain significant amounts of these oilseed proteins for their health benefits [3]. Efforts to improve taste/flavour of soy and peanutcontaining products could contribute positively to the utilization of these legumes in food [2]. The combination of improved technology, enhanced consumer health knowledge, and increased awareness about the health benefits of such products has the potential to increase the consumption of products containing soy and peanut [2].

Two primary interrelated factors have been associated with the off-flavours generated in soy and peanut containing products. These are the high proportion of unsaturated fatty acids especially linoleic acid and the presence of lipoxygenases [4]. When the lipid portion of these legumes are oxidized (enzymatic or nonenzymatic), they produce several compounds such as hydroperoxides, aldehydes, ketones, alcohols, lactones, aliphatic hydrocarbons and amines [5]. The carbonyls appear to be the most important compound due to its intense off flavour [5]. Lipoxygenases are of interest to the food scientist, due to their ability to form free radicals which can then attack other constituents and lead to the development of offflavours [6].

Heat treatments have been used to inactivate lipoxygenase and also remove some unwanted volatile compounds. Thermal treatment can range from minimal heat treatment (blanching), to cooking at atmospheric pressure and temperature or at a high pressure and high temperature (autoclaving) [5,6].

Removal of unwanted components in legumes can also be effected by soaking. The extent of removal of unwanted components however depends on the soaking temperature, type of soaking medium, type of legume, length of soaking 
and the solubility characteristics of the soluble component. Solutions of salts $\left(\mathrm{NaHCO}_{3}\right)$ and alkali $(\mathrm{KOH}, \mathrm{NaOH})$ have been employed to improve cell membrane permeability to enhance the removal of unwanted components $[5,6]$.

Fermentation processes have also been used to improve the keeping and sensory quality of food for many years [8]. The acceptability of many raw materials (such as soy beans) is preferred when fermented. Lactic acid bacteria (LAB) and other microbial cultures have been used to produce an array of fermented milk products. Fermentation of peanut milk with $S$. salivarius ssp. thermophilus was reported to be more effective than $L$. delbrueckii ssp. bulgaricus in reducing hexenal content in the milk [9]. Hexenal is one of the compounds responsible for undesirable green/ beany flavour in peanut milk. Strains of L. acidophilus have been reported to be effective in using stachyose and raffinose as carbon sources, and also generate good flavour when used to ferment soy-bean milk [10]. The study employed three treatment methods; thermal treatment, chemical treatment and microbial treatment of oilseeds to develop a yoghurt product from a vegetable milk blend. Proximate analysis was carried out on the product. The product was also sensorially analysed and validated to determine the optimum amounts of soy, peanut and cow milk required to develop a product that is appealing and acceptable to consumers, and still contain significant amounts of these oilseed proteins for health benefit.

\section{Material and Methods}

\subsection{Materials}

Red-skinned peanut seeds (Chinese variety) and soya bean seeds (Jenguma variety) were purchased from a registered seed grower in the Northern part of Ghana. Care was taken to ensure that good quality and mould-free seeds were selected. The starter culture (Lactobacillus bulgaricus, Streptococcus thermophilus) and cow milk used for study were obtained from Amrahia Dairy Farms, Amrahia in Accra, Ghana.

\subsection{Milk Preparation}

Sorted peanut seeds were blanched by submerging in boiling water $\left(100{ }^{\circ} \mathrm{C}\right)$ for 10 minute to inactivate the enzyme lypoxygenase known for its ability to cause oxidation which leads to the production of beany flavour [7, 11]. Peanut seeds required for the full fat yoghurt were then de-skinned and weighed before being soaked in $2 \%$ $\mathrm{NaHCO}_{3}$ for 18 hours. De-skinned peanut seeds to be defatted were flaked and weighed. The flakes were partially deffated by soaking in n-hexane for 18 hours in the ratio $1 \mathrm{~g}$ peanut seeds: $2 \mathrm{ml}$ hexane. The flakes were then washed with hot water $\left(70{ }^{\circ} \mathrm{C}\right)$. Soy beans were also steeped in boiling water $\left(100^{\circ} \mathrm{C}\right)$ for about 10 minutes, dehulled, weighed and then steeped in water for $16 \mathrm{hrs}$, and then in $2 \%$ $\mathrm{NaHCO}_{3}$ for 2 hours. Soaking in $\mathrm{NaHCO}_{3}$ was to soften the seeds and also remove the beany flavour as much as possible. The beans were then washed in hot water. The dehulled peanut and soya beans were separately mixed with water in a ratio of 1:5 [peanuts $(\mathrm{g})$ : water $(\mathrm{ml})$ ] and then milled to obtain the slurry. The slurry was filtered to obtain a smooth, fine, homogenized milk. Cow milk (0 - 2\%) was added to the prepared milk blends to obtain the formulations for the study.

\subsection{Mixture Design}

Ten milk formulations were processed into yoghurt by mixing the three basic ingredients; Full fat peanut milk/Partially defatted peanut milk, Soy milk and Cow milk. The proportions of these ingredients were obtained using a three component, constrained mixture design [11, 12]. Using design of experiments software, Minitab version 14, a mixture design (centroid design) was used to obtain 10 design points from the three components. The lower and upper bound constraints for each mixture component were used to generate the design (Table 1). The design was used to determine the optimum ratios of peanut milk, soy milk and cow milk that will yield the most acceptable product using sensory analysis (Table 2).

Table 1. Lower and upper limits of ingredients for formulation of vegetable milk yoghurt.

\begin{tabular}{ccc}
\hline Component & Lower limit & Upper limit \\
\hline Soy milk & 0.6 & 0.8 \\
Peanut milk & 0.2 & 0.4 \\
Cow milk & 0.0 & 0.2 \\
\hline
\end{tabular}

Table 2. Design matrix for ingredient formulations of vegetable milk yoghurt.

\begin{tabular}{ccccc}
\hline Formulation & Soy milk & $\begin{array}{c}\text { Peanut } \\
\text { milk }\end{array}$ & $\begin{array}{c}\text { Cow } \\
\text { milk }\end{array}$ & Total \\
\hline F1 & 0.60 & 0.40 & 0.00 & 1 \\
F2 & 0.70 & 0.20 & 0.10 & 1 \\
F3 & 0.63 & 0.33 & 0.03 & 1 \\
F4 & 0.60 & 0.20 & 0.20 & 1 \\
F5 & 0.80 & 0.20 & 0.00 & 1 \\
F6 & 0.63 & 0.23 & 0.13 & 1 \\
F7 & 0.70 & 0.30 & 0.00 & 1 \\
F8 & 0.73 & 0.23 & 0.03 & 1 \\
F9 & 0.60 & 0.30 & 0.10 & 1 \\
F10 & 0.67 & 0.27 & 0.07 & 1 \\
\hline
\end{tabular}

\subsection{Yoghurt Preparation, Nutritional and Sensory Evaluation}

\subsubsection{Starter Culture Preparation}

Freeze-dried yoghurt starter cultures of $S$. thermophilus and L. bulgaricus were obtained and revived separately in $12 \mathrm{~g} / 100 \mathrm{~g}$ sterilized milk broth and then transferred to the full fat peanut-soy-cow milk/partially defatted peanut-soycow milk broth for yoghurt production.

\subsubsection{Preparation of Peanut-Soy Milk Yoghurt}

Full fat peanut-soy milk/Partially defatted peanut-soy milk yoghurt was prepared by modifying a method reported earlier by Isanga and Zhang (2009). Each milk formulation was mixed and warmed at $43^{\circ} \mathrm{C}$ for 30 minutes. The milk 
was homogenized and pasteurized at $85^{\circ} \mathrm{C}$ for 30 minutes. The pasteurized milk was cooled to $43^{\circ} \mathrm{C}$ in a water bath and then inoculated with $3 \mathrm{ml}$ starter culture (L. bulgaricus and $S$. thermophilus; $1: 1)$ per $100 \mathrm{ml}$ milk. The mixture was incubated at $43^{\circ} \mathrm{C}$ for $3-4$ hours. At the end of the incubation period, the yoghurt was cooled and then transferred to a refrigerator at $\sim 5^{\circ} \mathrm{C}$ where it was stored overnight prior to analysis.

\subsubsection{Nutritional Characterization}

Proximate analysis was done on ten full fat peanut-soy milk yoghurt formulations, ten partially defatted peanut-soy milk yoghurt formulations and the control- cow milk yoghurt. Total solids, total nitrogen and ash were determined according to AOAC methods 925.09, 920.105 and 923.03 respectively [13]. Protein was calculated from total nitrogen using the conversion factors 6.25 and 6.38 for the vegetable milk yoghurt and cow milk yoghurt respectively. Fat content was determined by the Gerber method [14]. Carbohydrate was determined by difference. The calorific values were calculated using the expression:

$\mathrm{EV}(\mathrm{Kj} / 100 \mathrm{~g})=[(\% \mathrm{AC}$ X 17 $)+(\% \mathrm{P} \mathrm{X} \mathrm{17)}+(\% \mathrm{~F} \mathrm{X} \mathrm{37)}]$ where: $\mathrm{EV}=$ Energy value of food; $\% \mathrm{AC}=$ Percentage available carbohydrates; $\% P=$ Percentage protein; $\% F=$ Percentage fat [15].

\subsubsection{Sensory Evaluation}

Yoghurt samples were analyzed for appearance/color, texture/mouth feel, flavour and overall acceptability after overnight storage at $\sim 5{ }^{\circ} \mathrm{C}$. A total of sixty (60) untrained panelists were recruited from the Department of Nutrition and Food Science, University of Ghana, Legon. The criteria for recruitment were that they were yoghurt eaters and willing to participate in the test. The test was carried out in a well illuminated room free of environmental factors that could interfere with the normal perception of consumers.

The yoghurt samples included the 10 different specific ratios of full fat peanut-soy milk yoghurt in one set of experiment and the 10 different formulations of partially defatted peanut-soy milk yoghurt in another set of experiment. Consumers $(n=15)$ were asked to evaluate the 10 FPSY formulations on a 9 - point hedonic scale $(1-$ like extremely, 5 - neither like nor dislike and $9-$ dislike extremely) in an experiment. Consumers $(n=15)$ also evaluated the sensory attributes of the 10 DPSY formulations in another set of experiment. The samples were rated using a nine points hedonic scale with $1=$ like extremely, $5=$ neither like nor dislike and $9=$ dislike extremely. Since over six samples were tested in each experiment, a balanced incomplete block design (BIBD) was used to assign the samples to the panelists [16]. The following test design was used: $\mathrm{t}=10, \mathrm{k}=4, \mathrm{r}=6, \mathrm{~b}=15$, $\mathrm{N}=60, \lambda=2$.

\subsection{Optimization of Formulations}

Formulations that were rated within restricted ranges were used to generate the optimum region for each set of yoghurt formulation (FPSY and DPSY). Acceptable areas of the contour plots were superimposed to obtain the optimum region of formulations that will best appeal to consumers. For FPSY: Appearance (2 to 3), Taste (3 to 3.7), Flavour (3.2 to 3.7), Mouth feel (3 to 3.5) and Overall acceptability (3 to 4). For DPSY: Appearance (2 to 3), Taste (2.5 to 3.7), Flavour (2.5 to 3.5), Mouth-feel (3 to 3.5) and Overall acceptability (2.5 to 3.5$)$.

\subsection{Validation of Formulations}

To validate optimum formulations that appeal to most consumers, FPSY formulations were selected from the optimum region and areas outside the optimum region and presented to 15 consumers to evaluate in terms of appearance, taste, flavour, mouthfeel and overall acceptability. DPSY formulations were also selected from the optimum region and areas outside the optimum region and presented to 15 consumers in another set of experiment to evaluate in terms of appearance, taste, flavour, mouthfeel and overall acceptability. FPSY and DPSY formulations used for validation experiment are presented in Tables 3 and 4 respectively.

Table 3. FPSY formulations used for validation experiment.

\begin{tabular}{cccc}
\hline Formulation & Soy-Milk & Peanut Milk & Cow Milk \\
\hline 1 & 0.70 & 0.24 & 0.06 \\
2 & 0.68 & 0.25 & 0.07 \\
$3 *$ & 0.63 & 0.35 & 0.02 \\
$4^{*}$ & 0.74 & 0.25 & 0.01 \\
\hline
\end{tabular}

*Formulations outside optimum region.

Table 1. DPSY formulations used for validation experiment.

\begin{tabular}{cccc}
\hline Formulation & Soy-Milk & $\begin{array}{c}\text { Defatted } \\
\text { Peanut Milk }\end{array}$ & Cow Milk \\
\hline 1 & 0.65 & 0.22 & 0.13 \\
2 & 0.63 & 0.22 & 0.15 \\
$3^{*}$ & 0.73 & 0.25 & 0.02 \\
$4^{*}$ & 0.63 & 0.34 & 0.03 \\
\hline
\end{tabular}

*Formulations outside optimum region.

\section{Results and Discussion}

\subsection{Nutritional Characteristics of Partially Defatted Peanut-Soy Milk Yoghurt (DPSY) and Full Fat Peanut-Soy Milk Yoghurt (FPSY)}

The total solid content of partially defatted peanut-soy milk yoghurt (DPSY) samples ranged from 9.58\% - 11.23\% and were generally higher than that for full fat peanut soy milk formulations (FPSY) $(8.98 \%-10.62 \%)$ and lower than the control - cow milk yoghurt (13.80\%). Reduction in fat might have provided a suitable environment for increased fermentation in the defatted product. Decreased milk fat content subsequently decreases the initial $\mathrm{pH}$ of samples during fermentation [17]. Since lactic acid fermentation leads to an increase in the acid content of products it can be inferred that fermentation was facilitated in low fat milk as compared to high fat milk. Increased 
levels of defatted peanut milk and cow milk content in samples also increased the total solid content of samples (Figure 1).

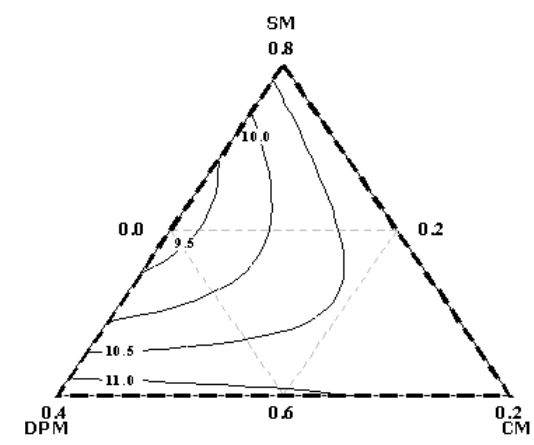

Figure 1. Mixture contour plot of total solids of DPSY formulations.

Crude protein content of DPSY ranged from $1.77 \%$ to $2.55 \%$ and was lower than FPSY $(2.18 \%$ and $2.91 \%)$. Crude protein content in the defatted product increased with increasing soy milk content and decreasing deffated peanut milk content (Figure 2). Peanut seeds were treated with hexane and subsequently washed with hot water $\left(100^{\circ} \mathrm{C}\right)$ to volatilize hexane present in the defatted seeds. Solvent treatment and subsequent hot water treatment of the oil seeds might have leached out some proteins from the peanut seeds resulting in the low crude protein values obtained in the defatted product. Hexane used in defatting improved cell membrane permeability, but removal of the unwanted components in the oilseed might have been accompanied by some loss in desirable nutrients such as proteins and minerals [5]. Generally the samples without cow milk had low crude protein content.

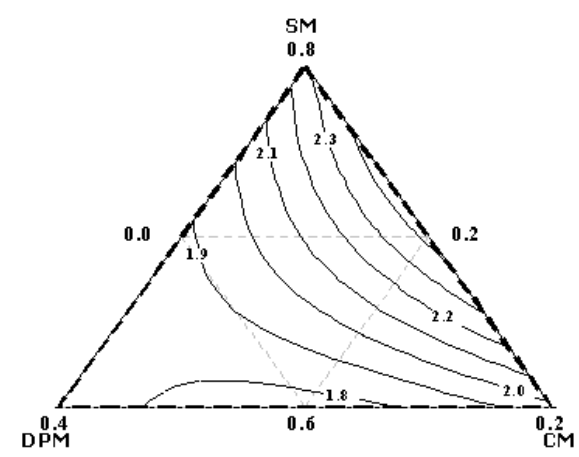

Figure 2. Mixture contour plot of crude protein content of DPSY formulations.

Carbohydrate content of the defatted product although recorded lower values than the control were generally higher than FPSY samples. Increase in defatted milk content increased the carbohydrate value of DPSY formulations.

Fat content of FPSY formulations was investigated and it ranged from 4.15 to $6.15 \%$. Fat content in the DPSY samples ranged between $2.10 \%$ and $3.00 \%$. However the fat content of cow milk yoghurt (control) was $2.48 \%$. Peanut milk influenced the fat content of the FPSY relative to the other two components (soy milk and cow milk). Formulations which had high peanut content recorded the highest percentage fat. The fat content of the defatted products as expected were lower than FPSY formulations and generally significantly higher than cow milk yoghurt ( $p$ $<0.05$ ). Aside this, the energy values of the defatted products were also lower than that of the FPSY samples. Since peanut seeds are known for their high fat content and thus high energy value, a decrease in fat content expectedly resulted in a decrease in energy value of the defatted peanut -soy milk formulations

Ash content of DPSY samples was significantly lower than the control (Cow milk yoghurt). The ash content of cow milk and cow milk yoghurt have been noted to be significantly higher than that of peanut milk and peanut milk yoghurt [15]. Ash content of DPSY samples increased with increasing cow milk content.

\subsection{Sensory Characteristics of Partially Defatted Peanut- Soy Milk Yoghurt (DPSY) and Full Fat Peanut-Soy Milk Yoghurt (FPSY)}

Sensory evaluation is a critical stage in product development and product optimization studies, because products targeted at consumers must first appeal sensorially to them. The sensory attributes of full fat peanut-soy milk yoghurt (FPSY) and partially defatted peanut-soy milk yoghurt (DPSY) were evaluated. Mean consumer scores for appearance, flavour, taste, mouthfeel and overall acceptability of FPSY and DPSY were determined (Tables 5 and 6). Mean scores for appearance, taste and flavour of FPSY formulations generally ranged from 2.00 (like very much) to 4.17 (like slightly) for appearance; 1.83 (like extremely) to 4.17 (like slightly) for taste; and 2.00 (like very much) to 4.67 (like slightly) for flavour. As regards the partially defatted product (DPSY) consumer acceptability scores ranged from 1.67 (like extremely) to 4.67 (like slightly) for appearance; 2.50 (like very much) to 4.83 (like slightly) for taste and for flavour scores ranged from 2.00 (like very much) to 4.17 (like slightly).

Table 5. Mean consumer scores for appearance, taste, flavour, mouthfeel and overall acceptability of FPSY.

\begin{tabular}{cccccc}
\hline Formulation & $\begin{array}{c}\text { Composition(\%) } \\
\text { SM:PM:CM* }\end{array}$ & Appearance & Taste & Flavour & Mouth feel \\
acceptability
\end{tabular}




\begin{tabular}{ccccccc}
\hline Formulation & $\begin{array}{c}\text { Composition(\%) } \\
\text { SM:PM:CM* }\end{array}$ & Appearance & Taste & Flavour & Mouth feel & $\begin{array}{c}\text { Overall } \\
\text { acceptability }\end{array}$ \\
\hline F6 & $63: 23: 13$ & $2.00 \pm 1.26$ & $2.83 \pm 0.75$ & $4.00 \pm 2.28$ & $2.33 \pm 0.81$ & $3.33 \pm 1.63$ \\
F7 & $70: 30: 0$ & $3.67 \pm 1.36$ & $4.17 \pm 2.48$ & $4.67 \pm 1.21$ & $5.00 \pm 2.75$ & $5.33 \pm 2.06$ \\
F8 & $73: 23: 3$ & $2.50 \pm 0.83$ & $1.83 \pm 0.75$ & $2.00 \pm 0.63$ & $2.17 \pm 0.75$ & $2.17 \pm 0.75$ \\
F9 & $60: 30: 10$ & $2.50 \pm 1.38$ & $3.67 \pm 1.51$ & $3.67 \pm 1.37$ & $2.83 \pm 0.75$ & $3.33 \pm 1.21$ \\
F10 & $67: 27: 7$ & $2.33 \pm 1.03$ & $3.33 \pm 1.96$ & $3.50 \pm 1.76$ & $4.00 \pm 1.09$ & $3.50 \pm 1.64$ \\
\hline
\end{tabular}

*Percentage proportion of soy milk: peanut milk: cow milk in mixture. A 9-point hedonic scale was used (1- like extremely, 5 - neither like nor dislike and 9 - dislike extremely). Values are means \pm standard deviations of duplicate determinations.

Table 6. Mean consumer scores for appearance, taste, flavour, mouthfeel and overall acceptability of DPSY.

\begin{tabular}{ccccccc}
\hline Formulation & $\begin{array}{c}\text { Composition(\%) } \\
\text { SM:DPM:CM* }\end{array}$ & Appearance & Taste & Flavour & Mouth feel & $\begin{array}{c}\text { Overall } \\
\text { acceptability }\end{array}$ \\
\hline F1 & $60: 40: 0$ & $2.00 \pm 0.63$ & $4.83 \pm 2.31$ & $3.50 \pm 0.83$ & $5.50 \pm 1.51$ & $4.66 \pm 1.86$ \\
F2 & $70: 20: 10$ & $2.50 \pm 0.54$ & $4.00 \pm 2.00$ & $2.83 \pm 1.83$ & $4.33 \pm 1.63$ & $3.66 \pm 2.06$ \\
F3 & $63: 33: 3$ & $4.00 \pm 1.54$ & $4.00 \pm 1.41$ & $4.16 \pm 1.94$ & $4.83 \pm 2.04$ & $4.50 \pm 1.51$ \\
F4 & $60: 20: 20$ & $1.83 \pm 0.75$ & $3.16 \pm 1.60$ & $2.83 \pm 1.32$ & $3.33 \pm 1.03$ & $3.16 \pm 1.32$ \\
F5 & $80: 20: 0$ & $4.66 \pm 1.50$ & $4.66 \pm 2.06$ & $4.16 \pm 1.60$ & $4.66 \pm 2.16$ & $3.83 \pm 1.16$ \\
F6 & $63: 23: 13$ & $1.66 \pm 0.51$ & $2.83 \pm 0.98$ & $2.00 \pm 0.89$ & $3.00 \pm 0.63$ & $2.50 \pm 0.83$ \\
F7 & $70: 30: 0$ & $3.66 \pm 1.75$ & $4.00 \pm 1.54$ & $3.33 \pm 1.03$ & $4.50 \pm 1.64$ & $4.50 \pm 1.64$ \\
F8 & $73: 23: 3$ & $3.00 \pm 1.67$ & $2.50 \pm 1.51$ & $2.50 \pm 1.87$ & $2.33 \pm 1.50$ & $2.83 \pm 1.32$ \\
F9 & $60: 30: 10$ & $3.50 \pm 1.04$ & $4.16 \pm 1.94$ & $3.33 \pm 1.63$ & $4.33 \pm 2.33$ & $4.16 \pm 2.04$ \\
F10 & $67: 27: 7$ & $4.00 \pm 2.44$ & $3.50 \pm 1.04$ & $3.66 \pm 1.75$ & $4.16 \pm 1.47$ & $3.66 \pm 1.03$ \\
\hline
\end{tabular}

*Percentage proportion of soy milk: defatted peanut milk: cow milk in mixture. A 9-point hedonic scale was used (1- like extremely, 5 - neither like nor dislike and 9 - dislike extremely). Values are means \pm standard deviations of duplicate determinations.

Acceptability of appearance, flavour and mouthfeel of FPSY increased as proportions of soy and peanut milk in the samples decreased. However consumer acceptability ratting for the taste of FPSY showed that consumer preference increased as the proportion of soy milk in the mixture increased. The overall acceptability of FPSY increased as soy milk and cow milk increased in formulations (Figure 3). Mixture contour plots for all the sensory attributes evaluated for the defatted product (DPSY) showed that as the proportion of defatted peanut milk increased in mixtures, consumer preference decreased. The overall acceptability of DPSY decreased as partially defatted peanut milk increased in samples and however increased with increasing soy milk and cow milk content (Figure 4). Consumers preferred products that had high cow milk content in the DPSY formulations.

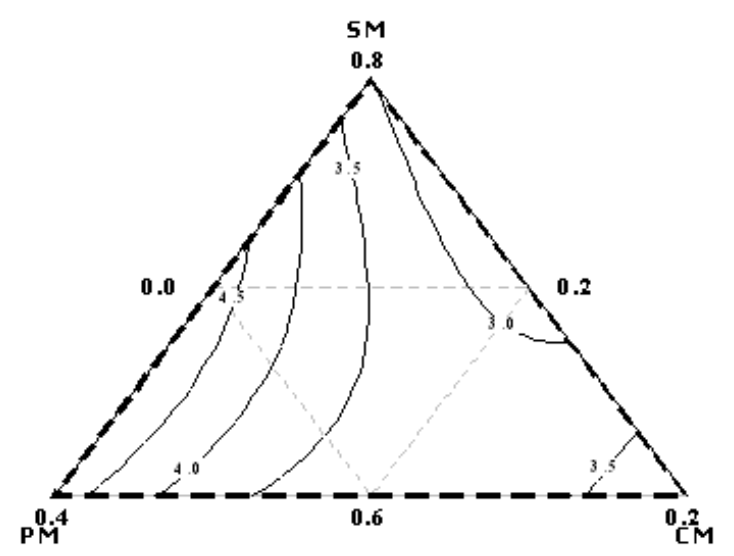

Figure 3. Mixture contour plot of overall acceptability of FPSY.

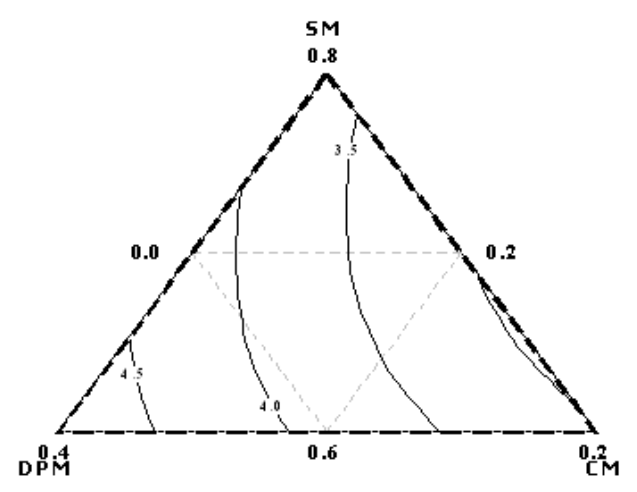

Figure 4. Mixture contour plot of overall acceptability of DPSY formulations.

\subsection{Optimized Formulations}

Optimization has been defined as a process of deriving the best of formulations amongst several [18]. When the acceptable areas of the contour plots were superimposed, Figures 5 and 6 were obtained for FPSY and DPSY respectively. From the contour plots of the optimized formulation, results for full fat peanut-soy milk yoghurt formulations show that the optimized region (portion in white) was within areas that had high soy milk content relative to cow milk and peanut milk proportions. However considering the optimized plot for the defatted product (DPSY), the optimized region skewed more towards high cow milk content relative to soy milk and peanut milk. This implies that while consumers preferred more soy milk in their full fat vegetable milk yoghurts, they preferred more cow milk in their low fat vegetable milk yoghurts to compensate for the loss fat. 


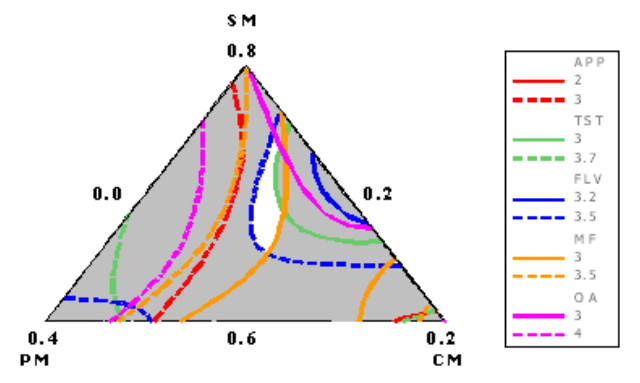

Figure 5. Optimized formulation for full fat peanut-soy milk yoghurt (FPSY).

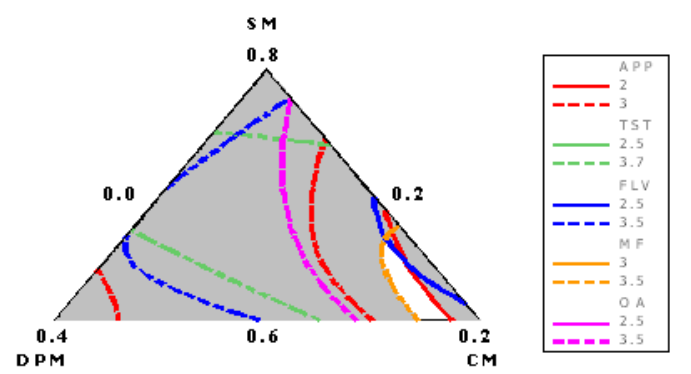

Figure 6. Optimized formulation for partially defatted peanut-soy milk yoghurt (DPSY).

\subsection{Validated Formulations}

The process of validating formulations in product optimization studies is to confirm products from the optimum region as the best formulations that appeal to most consumers contrary to points outside the optimum region. Formulations within the optimum region for FPSY formulation (Table 7) fell within the set (predicted) scores for the sensory attributes: Appearance (2 to 3), Taste (3 to 3.7), Flavour (3.2 to 3.7), Mouthfeel (3 to 3.5) and Overall acceptability (3 to 4). Formulations within the optimum region for DPSY formulation (Table 8) fell within the set (predicted) scores for the sensory attributes: Appearance (2 to 3 ), Taste (2.5 to 3.7$)$, Flavour (2.5 to 3.5$)$, Mouthfeel (3 to 3.5$)$ and Overall acceptability (2.5 to 3.5$)$.

Table 7. Mean scores of sensory attributes of validated FPSY formulations.

\begin{tabular}{cccccc}
\hline F & Appearance & Taste & Flavour & Mouthfeel & $\begin{array}{c}\text { Overall } \\
\text { acceptability }\end{array}$ \\
\hline 1 & 2.20 & 3.40 & 2.40 & 2.30 & 2.60 \\
2 & 2.20 & 2.80 & 2.30 & 2.40 & 2.00 \\
$3^{*}$ & 3.30 & 5.30 & 3.70 & 4.40 & 3.90 \\
$4 *$ & 4.20 & 5.11 & 5.30 & 5.00 & 5.10 \\
\hline
\end{tabular}

*Formulations outside optimum region. F - Formulation

Table 8. Mean scores of sensory attributes of validated DPSY formulations.

\begin{tabular}{cccccc}
\hline F & Appearance & Taste & Flavour & Mouthfeel & $\begin{array}{c}\text { Overall } \\
\text { acceptability }\end{array}$ \\
\hline 1 & 1.90 & 3.00 & 3.10 & 2.90 & 2.50 \\
2 & 1.90 & 3.50 & 3.40 & 3.20 & 3.10 \\
$3^{*}$ & 2.40 & 4.60 & 4.70 & 4.20 & 4.80 \\
$4 *$ & 2.70 & 4.70 & 4.80 & 4.40 & 4.80 \\
\hline
\end{tabular}

*Formulations outside optimum region. F - Formulation

\section{Conclusion}

Crude protein content and fat content of the partially defatted peanut-soy milk yoghurt formulations were lower than that of the full fat peanut-soy milk yoghurt. However the partially defatted yoghurt formulations had high carbohydrate and total solids content. Consumers preferred more soy milk in their full fat vegetable milk yoghurts but however preferred more cow milk in their low fat vegetable milk yoghurts. Optimized plots showed that the optimum region for FPSY was within areas that had high soy milk content relative to cow milk and peanut milk proportions, whiles the optimum region for the defatted product (DPSY) was skewed more towards high cow milk content relative to soy milk and peanut milk. Full fat peanut-soy milk yoghurt formulation with the most preferred sensory attributes consisted of 0.68 Soy milk, 0.25 Peanut milk and 0.07 Cow milk whereas partially defatted peanut-soy milk yoghurt with the most preferred sensory attributes was 0.65 Soy milk, 0.22 Defatted peanut milk and 0.13 Cow milk.

\section{References}

[1] Schmidt RH, Bates RP 1976. Sensory acceptance of fruit flavoured oilseed milk formulations. Proc. Fla. State Hort. Soc. $89,217-219$.

[2] Wu Y, Pope JF, Reagan S 2005. Attitudes and acceptability of soy-based yoghurt. In Yazici F, Alvarez VB, Hansen PMT 1997. Fermentation and properties of calcium-fortified soy milk yoghurt. J Food Sci. 62, 457 - 461.

[3] Drake MA, Gerard PD, Chen XQ 2000. Effects of sweetener, sweetener concentration, and fruit flavor on sensory properties of soy fortified yoghurt. J Sens Stud.16, $303-$ 405.

[4] Diamini AM, Mamba R, Silaula SM 2009. Attributes and consumer acceptance of yoghurt flavoured with noncultivated Indigenous Swazi Fruits. Afric J Food Agri, Nutr Dev. $9,636-649$.

[5] Sathe SK, Deshpande SS, Salunkhe DK 1984. Dry beans of Phaseolus. A review. Part 1. Chemical composition: Proteins. CRC Crit Rev Food Sci and Nutr. 20, 1-46.

[6] Robinson DS, Wu Z, Domoney C, Casey R 1994. Lipoxygenases and quality of foods. Food Chem. 54, $33-43$.

[7] Aidoo H, Sakyi-Dawson E, Tano-Debrah K, Saalia, FK 2010. Development and characterization of dehydrated peanut-cowpea milk powder for use as a dairy milk substitute in chocolate manufacture. Food Res Int. 43, 79-85

[8] Caplice E, Fitzgerald GF 1999. Food fermentations: role of microorganisms in food production and preservation. Int $\mathrm{J}$ Food Micro. 50, 131-149.

[9] Lee C, Beuchat LR 1991. Changes in chemical composition and sensory qualities of peanut milk fermented with lactic acid bacteria. Int J Food Micro. 13, 273 - 283.

[10] Kanda H, Wang HL, Hesseltine CW, Warner K 1976. Yoghurt production by Lactobacillus fermentation of soybean milk. Proc. Biochem. 1, 23 - 25. 
[11] Kpodo FM, Afoakwa EO, Amoa BB, Saalia FK, Budu AS 2013. Application of multiple component constraint mixture design for studying the effect of ingredient variations on the chemical composition and physico-chemical properties of soy-peanut-cow milk. Int Food Res J. 20, 811 - 818.

[12] Cornell JA 1983. How to run mixture experiments for product quality. American Society for quality control, Milwaukee, WI.

[13] AOAC 1990. Association of Official Analytical Chemists, $15^{\text {th }}$ edition, Washington, D.C.

[14] Pearson D 1976. The chemical analysis of foods. $7^{\text {th }}$ edition. Churchill Livingstone Edinburgh London. pp 402 - 452.
[15] Isanga J, Zhang G 2009. Production and evaluation of some physicochemical parameters of peanut milk yoghurt. Food Sci Tech. 6, 1132-2238.

[16] Cochran WG, Cox GM 1957. Experimental design. $2^{\text {nd }}$ edition. John Wiley and sons, New York.

[17] Shaker RR, Jumah RY, Abu-Jdayil 2000. Rheological properties of plain yoghurt during coagulation process: impact of fat content and preheat treatment of milk. J Food Eng. 44,175-180.

[18] Prinyawiwatkul W, Beuchat LR, McWatters KH, Phillips RD 1997. Optimizing acceptability of chicken nuggets containing fermented cowpea and peanut flours. J Food Sci. $62,889-901$. 